\title{
Papers
}

\section{Withholding the artificial administration of fluids and food from elderly patients with dementia: ethnographic study}

\author{
Anne-Mei The, Roeline Pasman, Bregje Onwuteaka-Philipsen, Miel Ribbe, Gerrit van der Wal
}

\begin{abstract}
Objective To clarify the practice of withholding the artificial administration of fluids and food from elderly patients with dementia in nursing homes. Design Qualitative, ethnographic study in two phases. Setting 10 wards in two nursing homes in the Netherlands.

Participants 35 patients with dementia, eight doctors, 43 nurses, and 32 families.

Results The clinical course of dementia was considered normal and was rarely reason to begin the artificial administration of fluids and food in advanced disease. Fluids and food seemed to be given mainly when there was an acute illness or a condition that needed medical treatment and which required hydration to be effective. The medical condition of the patient, the wishes of the family, and the interpretations of the patients' quality of life by their care providers were considered more important than living wills and policy agreements.

Conclusions Doctors' decisions about withholding the artificial administration of fluids and food from elderly patients with dementia are influenced more by the clinical course of the illness, the presumed quality of life of the patient, and the patient's medical condition than they are by advanced planning of care. In an attempt to understand the wishes of the patient doctors try to create the broadest possible basis for the decision making process and its outcome, mainly by involving the family.
\end{abstract}

\section{Introduction}

In 1997 there was widespread discussion in the Netherlands about the practice of withholding the artificial administration of fluids and food from patients with advanced dementia. This arose from a complaint about the staff of a nursing home who had deliberately allowed a patient with dementia to dehydrate. The patient's family had been told that this was common practice in the advanced stages of the disease. The family had the patient admitted to hospital, where he improved after an infusion of fluid. A few days later he was reportedly sitting up in bed and eating.

Withholding the artificial administration of fluids and food, especially in incompetent patients in nursing homes, has also been the subject of discussion in the United Kingdom and the United States. ${ }^{1-4}$ Whether the artificial administration of fluids and food is beneficial to patients with advanced dementia has been debated..$^{5-8}$

As a result of the debate in the Netherlands, the minister of public health, welfare, and sports commissioned this study. We aimed to determine the decision making process behind withholding the artificial administration of fluids and food in incompetent patients. ${ }^{9}$ Within this category, patients with dementia are an important group; $47 \%$ of people over 85 years have Alzheimer's disease. ${ }^{10}$ We focused on key factors in the decision making process, trajectories for the illness, advanced planning of care, and the presumed wishes of the patient.

\section{Methods}

Our qualitative ethnographic study was conducted in two phases. ${ }^{112}$ Data were collected through observation of the participants of two nursing homes. Informed consent was obtained from the management teams of the two homes, the doctors, the nursing staff, and representatives (mostly relatives) of the patients.

The first phase of the study (October 1998 to April 1999) was carried out by RP who worked full time for 7 months in a nursing home (201 beds) in a rural part of the eastern Netherlands. RP conducted formal interviews, attended patients' funerals, and interviewed a few of the bereaved families. The second phase of the study (December 1999 to February 2001) was carried out by AT in a nursing home (210 beds) in the more urbanised western Netherlands.

RP had investigated the practice of withholding the artificial administration of fluids and food by the staff full time for 7 months, but we found that the time frame was too short to understand decision making when illnesses had longer trajectories, and the attitudes of the other professionals were not sufficiently clear. Therefore we increased the study period for phase 2 to 14 months on a part time basis, 3 days a week. AT focused on the doctors and other healthcare professionals, the perspectives of all involved, formalities in planning care in advance (policy agreements and living wills), and the course of the illness long term. Retrospective information on prospective cases was
Department of
Social Medicine,
Institute for
Research in
Extramural
Medicine, Vrije
University Medical
Centre, 1081 BT,
Amsterdam,
Netherlands
Anne-Mei The
anthropologist
Roeline Pasman
sociologist
Bregje
Onwuteaka-Philipsen
health scientist
Gerrit van der Wal
professor
Department of
Nursing Home
Medicine, Institute
for Research in
Extramural
Medicine
Miel Ribbe
professor
Correspondence to:
A-M The
am.the.emgo@
med.vu.nl
bmj.com 2002;325:1326 
supplied from the medical notes and interviews. AT attended meetings, had informal conversations, and conducted interviews.

Because of the media attention surrounding the practice of withholding the artificial administration of fluids and food, the staff of both nursing homes were initially conscious of the researchers. The researchers observed but did not participate in the decision making process about withholding fluids and food. The findings and conclusions were submitted to the participants and discussed in formal interviews conducted at the end of both phases. ${ }^{13}$

Overall, 35 of the patients (28 women) on the 10 included wards were candidates for the withholding of the artificial administration of fluids and food, especially those with advanced disease. Their age ranged from 61 to 98 years. Four patients were in a ward for those needing supervision only, 12 in a ward for patients needing care for daily activities only, and 19 in a ward for patients needing nursing care. It was not possible for the researchers to follow patients on more than five wards, and the sample size was based on this observation. Eight doctors (five male, three female), 32 families, and 43 nurses were observed in the decision making process.

\begin{abstract}
Analysis
Our analysis and results are based on four types of data $^{13}$ : the researchers made comprehensive notes of their observations and informal conversations; formal interviews were taped and fully transcribed; the researchers had access to the medical and nursing records of the patients for which their representatives had given consent; and the researchers kept a diary of
\end{abstract}

\section{Box 1: Conversation with family of patient with dementia}

Doctor S: Please sit down $\mathrm{Mr} \mathrm{H}$. Your wife has been here now for four weeks. Let me start by asking you whether you have any questions. $\mathrm{Mr} \mathrm{H}$ : Well, doctor, what can I say? It's alright now. It's a great relief for me that she's in good hands here.

Doctor $\mathrm{S}$ : We have the idea that your wife is starting to get used to it now. Making the change is difficult, and she will have to settle down, but she's beginning to quieten down.

(The conversation continues with a discussion about the admission and the care plan that has been drawn up for Mrs $\mathrm{H}$.)

Doctor S: Let me make myself clear Mr H. I can't make your wife better. The dementia will progress, and she will slowly deteriorate. What we can do is to try and make her life here as comfortable as possible for her. And it's mainly the nursing staff who will do that for her.

$\mathrm{Mr} \mathrm{H}$ : That's alright, doctor.

Doctor S: Once every six months your wife's condition will be discussed in the multidisciplinary meeting. After that, I'll ring you up and invite you to come here to discuss things. Of course, if anything important happens in the meantime, we will contact you earlier. If you have any questions, you can always contact me. Together with you, we will try to look after your wife to the best of our ability. That's why good contact between us is very important. It's not yet necessary, but in the future we will, for instance, be faced with difficult decisions. The time will come when your wife will not be eating properly, either because she no longer recognises the food or because she has difficulty in swallowing, which means she will lose weight. There can then come a time when, for instance, we will have to decide whether we prolong her life by giving her food and fluid through a tube. $\mathrm{Mr} \mathrm{H}$ : Drip feeding?

Doctor S: Or giving her extra fluids. However, as I was saying, this is not yet necessary, and we hope that it will stay that way for a long time, but I want to mention it now, so that you already know. their own behaviour and attitudes, distinct from their comprehensive notes.

Data were analysed per patient by both researchers, resulting in 35 case studies, which contained detailed descriptions of illness trajectories, the decision making process, the participants, and communication between the participants. Completed case studies were read repeatedly to identify patterns. The cases studies were then compared and similarities and differences between topics analysed. ${ }^{14}$ We resolved discrepancies through discussion. Saturation of data was reached after 21 cases; the identified patterns were checked with the last 14 cases.

\section{Results}

\section{Advanced planning of care}

The family was involved from the moment a patient was admitted to the nursing home. This was achieved by holding regular meetings, starting shortly after admission, in which the deterioration and subsequent management of the patient were discussed (box 1).

Often these conversations resulted in policy agreements, which were recorded in the patient's notes. Few patients had a written living will; in specific situations they did not want their life prolonged or wanted euthanasia. These wishes were recorded.

\section{Illness processes}

Trajectory 1: the slow downward curve

When dehydration resulted from dementia there was hardly ever a decision to give fluids and food artificially. Slow deterioration was considered a natural course of the disease, in which patients would not benefit from the artificial administration of fluids or food. Patients were, however, given other types of non-medical support, including special dietary provisions, and extra attention was paid to the way food was given. This trajectory took place mainly in a care context whereas the tasks depended largely on the nursing staff.

The decisions to withhold fluids and food in this illness trajectory seldom led to problems: caregivers and family expected the slow deterioration because it could be seen and, from the conversations with the doctors, they were prepared (box 2). There was usually mutual agreement not to prolong the patient's life unnecessarily, because it was considered that hydration would not improve the quality of the patient's life and further exposure to the disease process.

\section{Trajectory 2: the interruption}

Acute illness such as an infection or depression often interrupted the slow deterioration from dementia (15 of 35 cases). This accelerated deterioration was usually considered to be reversible and medically treatable.

When acute illness was accompanied by dehydration, in most cases (15 times in nine patients) a decision was made to rehydrate the patient (14 times by hypodermoclysis in eight patients: one patient was treated four times, three patients twice, and four patients once). If fluids were given artificially this happened only in combination with medical treatment (box 3). The argument was that "if you say A, you've also got to say B." This trajectory was characterised by a medical context. 
In the case of brief acute illnesses or when the patient was in a poor condition or in end stage dementia, doctors were more reluctant to begin medical treatment, including the artificial administration of fluids and food. One doctor said that "patients are vulnerable-each period of treatment makes it more difficult to treat again. At a certain point it's no longer beneficial." In such situations the illness process was considered to be irreversible. If doctors anticipated this, they prepared the family by warning that more treatment wouldn't help, which was recorded in the patient's notes.

\section{Unexpected fluctuation in the illness process: the dynamics}

The decision to withhold the artificial administration of fluids and food was mainly dictated by the medical condition of the patient and the presence of acute illness. The course of the illness process, however, seemed difficult to predict. Some patients became suddenly ill and died, whereas those who were expected to die recovered unexpectedly, even after simple treatment (box 4). For individual patients various and even different decisions were made to withhold the artificial administration of fluids and food.

\section{Decision processes}

\section{Living will and policy agreements}

The patients were unable to make a choice for themselves about treatment, yet their wish was a factor in the decision making process. A written living will influenced the decision to withhold the artificial administration of fluids and food. Although euthanasia was not possible, the living will was considered to represent the patient's wish not to prolong his or her life, which was respected. Written agreements were considered useful for dialogue with families, subsequent conversations, and locums. Unexpected fluctuations in the patient's condition, however, influenced previous policy agreements; the doctors stated they mainly focused on the recent and current wellbeing (including prognosis) and quality of life of their patients.

\section{Current verbal and non-verbal wishes}

Some patients with early dementia clearly expressed their death wishes verbally, sometimes confirmed by the family (box 5). There also seemed to be non-verbal expressions of wishes; patients who gave the impression they were tired of life or even wanted to die-for example, by refusing food or drink (box 6).

A patient's behaviour and attitude was also considered by the staff to be a non-verbal expression; if giving the impression of being happy, this was interpreted as "a will to live." The opposite was also the case; if a patient's quality of life was poor for months despite drugs, the decision to not treat was made when they developed pneumonia with dehydration.

Although the ways in which a patient's wishes were expressed were recorded by the doctors, important unexpected changes did occur. For instance, one patient who was thought to have a death wish, got out of bed after a few weeks. She said nothing about wanting to die, and gave the impression of enjoying life. When she developed pneumonia a year later, it was decided together with her son not to keep to the former policy agreement but to treat her with antibiotics and hypodermoclysis.
Box 2: Conversation with the family about the onset of advanced dementia

Doctor L talking to Mrs J's daughter and son in law: It's already quite some time since we saw each other last.

Mrs J's daughter: We sometimes see each other in the corridor, doctor. Doctor L: Yes, of course. I mean sitting opposite each other and having a more official conversation. The reason is that last week we talked about your mother in the multidisciplinary meeting, and we think it would be a good idea to discuss a few things. Is there anything that you, yourselves, wish to discuss?

Mrs J's daughter: She's getting a lot worse. She really hasn't recognised us for some time now. And eating is getting more and more difficult, too. When I come here I always bring some sweets with me, because she loves them, but she just leaves them in her mouth. It's as if she can't swallow.

Doctor L: We notice that too. That's been going on now for some time. The last time we spoke to each other we decided to put your mother on a special diet. She now has all her hot meals minced up, and we're giving her extra protein. If she can't manage that, we give her liquid food.

Mrs J's daughter: Those packets. She loves the banana taste.

Doctor L: We've been able to keep that up for some time, but there's not really much more that we can do. And, in spite of everything, she continues to lose weight. It's not yet necessary, but the next step is to decide whether we are going to feed her artificially.

Mrs J's son in law: Through a tube?

Doctor L: For instance. But the question is is that the right thing to do, and is it going to help her?

Mrs J's daughter: No. My mother would never have wanted that. She always said that she didn't want to be kept alive. No, no tubes.

Doctor L: That's exactly what we think. If we thought that we would be helping your mother, for instance to get over a temporary relapse by feeding her artificially, we would certainly consider it. That was the case last year, when we gave her antibiotics because she had pneumonia. We also gave her a hypodermoclysis because she was dehydrated and the antibiotics would otherwise have been ineffective. She improved a lot after that. At least we think that she did quite well last year. But now we're faced with a different situation. Now the dementia has progressed, and there is no way of stopping it. We can try to slow down the process, as we have done by spoiling her, giving her all kinds of supplements in her food, and the treatment we gave her last year. But there comes an end to that process. Your mother is getting towards the end, and that's what we feel is happening now. This is the last phase that we are approaching-the phase of withering away.

Mrs J's daughter: We can see that too. It might sound a bit hard, but as far as I'm concerned she can close her eyes. All she does is to sit in that chair, and nothing else. A year ago I wouldn't have said that, but now I do.

Doctor L: Your mother is still here, but the real question is: what is her quality of life? On the other hand, we don't have the impression that she's suffering. And we'll do everything we can to prevent that. We will continue to provide the best possible care for her. We offer her food and drink, and try to spoil her as much as possible. I'll make a report of this discussion and record in the files that we have decided not to prolong the life of your mother unnecessarily, as they say in the medical terminology. If necessary, then we'll discuss the matter again. Just because it's written down, that doesn't mean that we will do it whatever happens. We can discuss each new situation when it occurs.

\section{Control of staff and adaptation of family}

The care context of the slow downward curve (trajectory 1) depended on the nursing staff. Against that the staff rarely participated in the decision making process for withholding the artificial administration of fluids and food; they were informed and their opinions sought, but the doctors made the decisions. Nurses influenced the doctors by questioning decisions and expressing their concerns.

The doctors' decisions were mostly influenced by their reaction to the patient's condition and the patient's family. The family had a considerable influence, formally as legal representatives; however, it was more their emotional response to the patient's 


\section{Box 3: Fluids given in combination with medical treatment}

Doctor R: I'm glad you could come so quickly. The situation has been getting a lot worse in the past few hours. Your mother is a diabetic, and the level of glucose in her blood fluctuates a lot. You know how much trouble we've had, since she was admitted three months ago, to regulate her glucose. But also, she's not eating or drinking properly. She has lost four kilos in a very short time. So we need to do something about it.

(Mrs M's daughter nods.)

Doctor R: A week ago she also got pneumonia, which we've given her antibiotics for. Since yesterday she's been deteriorating. She's dazed and is hardly eating anything at all. Today things have got worse again. She's barely conscious, but she does react when you talk to her.

Mrs M's daughter: With difficulty, doctor. I sit beside her bed and try to talk to her, but it's almost impossible. Of course, she's also almost blind and deaf, so that doesn't make it any easier.

Doctor R: The situation is this. She hasn't reacted well enough to the antibiotics, and therefore I want to prescribe another type of antibiotic. But that's not all. Because of the fluctuating glucose levels, she's dehydrated, and we will therefore have to give her extra fluids. Otherwise the antibiotics won't work and it will be useless to prescribe them for her. Let's put it this way: "If you say A, you've also got to say B."

(Mrs M's daughter nods again)

Doctor R: We give the fluid through small needles in the legs, and let it seep into the body that way. She will hardly feel anything at all. Just a little prick when we insert the needle. What I'm asking you now is whether you agree with this.

Mrs M's daughter: I certainly want to try it. She's not yet got to the stage that I would think "let her go." No absolutely not. She can be so well sometimes. Doctor R: Does she ever talk to you about how she experiences life? Mrs M's daughter: She doesn't want to die yet, she tells me. No, she doesn't want that.

Doctor R: Alright, then I'm going to arrange for her to be given the medicine and the fluids. doctors considered the wishes of the families, but in the end they had control over treatment.

\section{Discussion}

Patients with dementia follow two illness trajectories, which entail decisions about whether to withhold the artificial administration of fluids and food. Doctors in nursing homes consider the deterioration from dementia (trajectory 1) to be the normal, irreversible course of the disease. Therefore in advanced dementia it is rarely considered beneficial to give fluids and food artificially. In our study this usually only happened if there was an acute illness or a condition that needed medical treatment (trajectory 2 ) and required rehydration to be effective.

Care providers strove to comply with the wishes of their patient (reconstruction of patient's wish). ${ }^{15}$ Patients' living wills seemed of limited importance;

\section{Box 5: Verbal expression of wishes}

Researcher: Are there any patients who are eligible for my study?

Doctor: Mrs B is withering away in her bed. Researcher: Can you tell me something about her? Doctor: Mrs B was admitted because she was in need of care, and on admission it was already clear that she didn't want to live any longer. She had been wanting to die for a long time. Her sons told us about that. Mrs B is still capable to express her will. And she continues to express her death wish to us since she is here in our nursing home to me and to the nursing staff. Mrs B has a so called "repeated death wish." So we have a 97 year old person who had not wanted to be admitted to a nursing home and who already for a long time expresses the wish to die. I must say that I can understand it. We had many conversations with her oldest son in the past year. It has been decided that, in view of her present quality of life, life prolonging treatment is not indicated. For the past few weeks she's been eating and drinking less all the time. It seems to us that she doesn't want food or drink because she wants to die. We continue to offer it to her, but respect her wishes if she doesn't want it. Last week I said to her "if you don't eat or drink you will die," and she made clear that that was her wish. So what else can we do than respect this?

policy agreements were useful in the decision making process and for dialogue with the family. But in the end the medical condition of the patient, the wishes of the family (considerable attention was paid to helping the family adjust to the prevailing circumstances), and the interpretations of the patients' quality of life by their care providers were the most important criteria for withholding the artificial administration of fluids and food.

Doctors are constantly faced with uncertainties about what the patient wants (a question of interpretation), and therefore they struggle in deciding what is medically best. Unexpected fluctuations in the disease and presumed life wishes of patients force doctors to continue to ask themselves this question. To reduce this uncertainty they try to create the broadest possible basis for the decision making process and its outcome, mainly by involving the family. 


\section{What is already known on this topic}

Debate has focused on whether it is beneficial to withhold the artificial administration of fluids and food from patients with advanced dementia

\section{What this study adds}

The course of dementia, the patient's quality of life, and the patient's current medical condition influence doctors' decision making more than advanced planning of care

Doctors try to create the broadest possible basis for the decision making process and its outcome, mainly by involving the family

The two illness trajectories had different contexts and concerned different professionals: the second trajectory was characterised by medical decision making by the doctors and the first trajectory by care from the nursing staff. The care context seemed the most vulnerable: more staff were involved and the borderlines for what was involved in care were less clear. The care context is sensitive to continuity and communication problems; at present there is a scarcity of nurses in the Netherlands, which leads to unfilled posts and temporary workers.

\section{Methodological considerations}

We identified repeated patterns of decision making in the practice of withholding the artificial administration of fluids and food from elderly patients with dementia in nursing homes. We assume that our findings are generalisable because the patterns were observed in two nursing homes in different regions and by two researchers working independently. Further ethnographic research is needed to confirm the generalisability of our findings. ${ }^{11}$

The findings are important because the doctors' attitude towards policy agreements explained their preference for concentrating on the patient's quality of life. Although written living wills were helpful, they were insufficient. Because of the dynamic course of dementia, the current medical condition of the patient and his or her wishes must be taken into consideration when deciding whether to withhold the artificial administration of fluids and food.

Contributors: A-MT and RP were involved in the conception and the design of the study, collected data, contributed to the analysis and interpretation of the data, and wrote the paper. BP $\mathrm{GW}$, and MR were involved in the conception and design of the study and contributed to the analysis and interpretation of the data and the final version of the paper.

Funding: This study was funded by grants to the value of $€ 297000$ ( $£ 186932 ; \$ 293466$ ) from the Ministry of Health, Welfare, and Sports, the Netherlands.

Competing interests: None declared.

Sheldon T. Row over force-feeding of patients with Alzheimer disease BMJ 1997;315:327-32.

2 Kmietowicz Z. Woman dies two months after food withdrawal. BMJ 1997;314:1501.

3 Dyer C. Police investigate "euthanasia" deaths. BMJ 1999;318:143.

4 Dyer C. Withdrawal of food supplement judged as misconduct. $B M J$ 1999:318:895.

\section{Box 6: Non-verbal expression of wishes}

Mrs N, 87 years old, has Alzheimer's disease. After a month in the nursing home she regularly refuses to eat or drink. When she is given a plate of food she either forces it aside or tips it onto her neighbour's plate. When the nurses try to feed her, she turns her head away and closes her lips tightly. She refuses to talk to anyone. All kinds of tests are carried out to determine the underlying reason. The doctor examines her stomach. Together with the psychologist the doctor investigates whether it might be depression, the speech therapist examines her to find out whether she has difficulty in swallowing, and the nursing staff take a great deal of trouble to make her feel at home in the nursing home and find out from the family what her favourite food is. To enable the antidepressants to take effect, Mrs $\mathrm{N}$ has to be rehydrated by hypodermoclysis. The argument of the physician is that "if you say A, you've also got to say B."

Twice Mrs N pulls the needles out of her legs. The doctor and the nursing staff interpret this as a protest against the artificial administration of fluids. Therefore the doctor no longer continues with the hypodermoclysis. In spite of the antidepressants, Mrs N continues to refuse food and drink, which makes the nursing staff feel totally powerless. She dies two weeks later.

\section{Box 7: Decision making in favour of the family by locum doctor}

Mrs W is 87 years old and has been in the nursing home for three years. Her youngest daughter was inseparable from her, which had caused considerable tension, especially with the nursing staff. No written policy had been formulated for her. Mrs W became ill during the weekend, and the doctor on duty came to the nursing home.

Doctor: I decided to give Mrs W hypodermoclysis because this was the wish of the family. You see, when you're on duty it's a difficult situation. There was no recorded policy and I had never seen this woman before. Her daughter literally begged me to save her mother. She was obviously not ready to say goodbye to her mother. I did no harm to the woman by making this decision and, in my opinion, if the patient doesn't suffer then the family is a legitimate reason for taking such actions.

Researcher: But if you, yourself, had to make a decision and the daughter had not insisted in such a way, what would you have done?

Doctor: Just medically and looking at the patient no, I don't think I would have done it. But I'm not sure because I didn't have a relationship with this family so it was not clear in which phase of saying farewell they were. Like I said, I decided to give a hypodermoclysis because of the daughter, she needed definitely more time, and when you're on duty you're always more careful with family. For me, and I think for many colleagues, family is always very important in these kinds of decision making. You must not forget that they have to go on after their mother or father dies. Mrs W's daughter expressed her gratitude dramatically-she threw her arms around my neck and kept saying "thank you, doctor, thank you."

5 Gillick MR. Rethinking the role of tube feeding in patients with advanced dementia. N Engl J Med 1999;342:206-10.

6 Sheiman SL. Tube feeding the demented nursing home resident. J Am Geriatr Soc 1996;44:1268-70

7 Onwuteaka BD. Withholding artificial administration of fluids and food from elderly patients with dementia: a research proposal. Amsterdam: EMGO Institute, 1998.

8 Volicer L Is hospice care appropriate for Alzheimer patients? CARING Magazine Nov 1993;50-5.

9 Finunce TE, Christmas C, Travis K. Tubefeeding in patients with advanced dementia: a review of evidence. JAMA 1999;282:1365-70.

10 Huang ZB, Ahronheim JC. Nutrition and hydration in terminally ill patients. An update. Clin Geriatr Med 2000;16:313-35

11 Savage J. Ethnography and health care. BMJ 2000;321:1400-2.

12 Pope C, Mays N. Reaching the parts other methods cannot reach: an introduction to qualitative methods in health and health services research. $B M J$ 1995;311:42-5

13 Mays N, Pope C. Assessing quality in qualitative research BMJ 2000;320:50-2

14 Strauss A, Corbin J. Basics of qualitative research. Newbury Park: Sage, 1990

15 Ackerman TF. The moral implications of medical uncertainty: tube feeding demented patients. JAm Geriatr Soc 1996;44:1265-7.

(Accepted 31 May 2002) 